\title{
PERSPECTIVAS DE LA POBLACIÓN BENEFICIADA DEL PROGRAMA HÁBITAT INSTRUMENTADO EN LOS MUNICIPIOS DE KANASÍN Y UMÁN, YUCATÁN
}

\section{PERSPECTIVES FOR THE BENEFICIARIES OF THE HABITAT PROGRAMME IMPLEMENTED IN THE MUNICIPALITIES OF KANASIN AND UMAN, YUCATAN}

\section{Rafael Ortiz Pech Facultad de Economía Universidad Autónoma de Yucatán, México rafael.ortiz@uady.mx}

\section{Gerardo García García} Facultad de Medicina Verterinaria

Universidad Autónoma de Yucatán, México garcia@uady.mx
Yolanda Oliva Peña

Centro de Investigaciones Regionales

Universidad Autónoma

de Yucatán, México

opena@uady.mx

\section{RESUMEN}

El programa Hábitat se instrumentó a nivel federal desde 2003 como una forma de combatir la extrema pobreza y marginación en comunidades urbanas que se consideren en situaciones críticas. En este artículo se presenta el estudio de perspectiva de los usuarios de apoyos y cursos que se realizaron en áreas marginadas de Umán y Kanasín, en la zona llamada "metropolitana" de Mérida. Los resultados muestran que es necesario eslabonar programas de desarrollo social a fin de incidir en el abatimiento de la pobreza y marginación, ya que a partir de los datos obtenidos no es posible dar cuenta de cambios importantes en los niveles de pobreza.

Palabras clave: programa Hábitat, pobreza extrema, alta marginación, Kanasín y Umán

Clasificación JEL: I38, O18, R28, R58 


\section{ABSTRACT}

Habitat Program is implemented to federal level up to 2003 as a way to fight the extreme poverty and marginalization in communities (area) that is considered as critical situation. This article presents the user's perspectives of the implementation of supports and courses that was realized in that lagged areas like Uman and Kanasin within the so-called Metropolitan Zone of Merida. The results show that is necessary to chain programs of social development to diminish the poverty and marginalization since the obtained data don't confirm important changes in the level of poverty.

Keywords: Habitat program, extreme poverty, marginalization, Kanasin and Uman 


\section{INTRODUCCIÓN}

La crisis económica mundial que afecta a México, junto con los problemas estructurales generados por el modelo económico y político, han traído como consecuencia la profundización del empobrecimiento de importantes segmentos sociales; por lo que el tema del desarrollo económico y la pobreza son algunas de las prioridades que los gobiernos deben atender.

Pese a los esfuerzos realizados en el país, el índice de pobreza extrema y marginación se ha incrementado en los últimos años, lo que hace necesario la evaluación de la política pública en los tres órdenes de gobierno.

A nivel estatal, Yucatán presenta problemas de alta marginación y pobreza extrema en amplios sectores de población, aunque aplican programas que intentan superarlos. Por tal motivo, en este artículo se estudian los municipios de Kanasín y Umán, que pertenecen a la llamada zona metropolitana de Mérida, para describir cuales son las perspectivas de los usuarios del programa Hábitat en su área de influencia.

Para ello se precisa, cómo es posible definir el concepto de pobreza y marginación y los distintos enfoques que se utilizan para contextualizarlo con este tipo de programa cuando se instrumenta en los lugares urbanos (polígonos) donde se propone coadyuvar a la resolución de problemas socioeconómicos.

Así, se presentan los resultados obtenidos a través de encuestas aplicadas a hogares que han participado dentro del programa Hábitat, lo que permite conocer su percepción acerca de los beneficios recibidos entre las familias beneficiarias, ventajas, opciones y posibles razones de la falta de eficacia. De este modo contribuye a entender el funcionamiento del programa desde la perspectiva del usuario, pues utiliza información de fuentes primarias y expone los resultados para el combate la pobreza extrema y la alta marginación. 


\section{ANTECEDENTES}

En México el programa Hábitat se instrumenta a partir del año 2003 por la Secretaría de Desarrollo Social (SEDESOL) a través de subsidios asignados por el gobierno federal. El programa surge orientado al combate a la pobreza, marginación e inseguridad pública por medio del mejoramiento de la infraestructura urbana y el desarrollo de las capacidades comunitarias e individuales, entre otros (SEDESOL, 2012). Este apoyo está condicionado a toda localidad que no supere los 15,000 habitantes, contar con al menos $80 \%$ de los lotes o viviendas ocupadas, que tenga déficit en infraestructura física y que físicamente no se encuentre dentro de zonas ecológicas. Cada año se realiza una selección de áreas de operación del programa conocidas como polígonos Hábitat, que son, prácticamente, zonas urbanas marginadas.

Según SEDESOL, el programa Hábitat cuenta con varios tipos de apoyo cuyos recursos son de origen federal y local, presentado en tres modalidades. El primero, llamado desarrollo social y comunitario, tiene como objetivos impulsar las capacidades individuales y comunitarias, fomentar la organización y participación, apoyar a personas mayores para que transmitan sus conocimientos, experiencias, habilidades y vocación a la comunidad; entre otros. La segunda, el mejoramiento del entorno urbano, apoya obras y acciones para el mejoramiento en infraestructura urbana básica, como agua potable, electricidad, alumbrado público, calles, adoquines, etc. También previene riesgos en los asentamientos humanos y capacita a la población que se encuentra en pobreza extrema en el tema ambiental.

La tercera modalidad es la promoción del desarrollo urbano, cuyo propósito es la elaboración y actualización de planes y programas municipales de desarrollo urbano y territorial (como los planes de ordenamiento territoriales municipales). Incluye también la elaboración de estudios que ayuden a superar la pobreza urbana en los polígonos definidos a través de la capacitación, entre otros. 


\section{Cuadro 1}

Estadísticas principales del programa Hábitat

\begin{tabular}{|l|r|r|}
\hline \multicolumn{1}{|c|}{ Categoría } & \multicolumn{1}{|c|}{2006} & \multicolumn{1}{|c|}{2008} \\
\hline $\begin{array}{l}\text { Recursos ejercidos (en millones de } \\
\text { pesos) }\end{array}$ & $\$ 1,992$ & $\$ 1,887$ \\
\hline Ciudades y ZM* elegidas & 205 & 245 \\
\hline Municipios elegidos & 267 & 353 \\
\hline Polígonos atendidos & 992 & 1,173 \\
\hline Proyectos apoyados & 10,535 & 8,181 \\
\hline
\end{tabular}

Fuente: SEDESOL (2006, 2012), *ZM= Zonas metropolitanas

Para poder cumplir con los objetivos planteados en el programa se han erogado diversos subsidios de manera creciente (cuadro 1). En el año 2006 a nivel federal se destinaron 1,992.1 millones; adicionalmente se complementó con otros fondos estatales, municipales y por los propios beneficiarios (SEDESOL, 2006), beneficiándose a 715,000 hogares declarados en pobreza patrimonial.

Según cifras de SEDESOL $(2008,2009)$ en el estado de Yucatán el total de hogares beneficiados del programa ha disminuido; mientras que se observa un aumento en el número de polígonos Hábitat en casi 15\% (cuadro 2).

\section{Cuadro 2}

Beneficiados del programa Hábitat en Yucatán (2008-2009)

\begin{tabular}{|l|c|c|}
\hline \multicolumn{1}{|c|}{ Concepto } & 2008 & 2009 \\
\hline Total hogares beneficiados & 56,265 & 42,011 \\
\hline Hogares pobres beneficiados & 30,945 & 30,757 \\
\hline Polígonos Hábitat & 52,393 & 60,225 \\
\hline
\end{tabular}

Fuente: SEDESOL $(2008,2009)$.

Al analizar lo anterior, para Kanasín y Umán, se tiene que solamente hay información disponible para el año 2008 y se observa que son muy similares los datos de beneficiados (cuadro 3). 


\section{Cuadro 3}

Beneficiados del programa Hábitat en Kanasín y Umán (2008)

\begin{tabular}{|l|c|c|}
\hline \multicolumn{1}{|c|}{ Concepto } & Kanasin & Umán \\
\hline Hogares en polígonos Hábitat & 3,907 & 3,451 \\
\hline Hogares pobres en polígonos Hábitat & 2,520 & 2,206 \\
\hline Total hogares beneficiados & 3,362 & 3,451 \\
\hline Hogares pobres beneficiados & 2,690 & 2,206 \\
\hline
\end{tabular}

Fuente: SEDESOL (2008).

Otro aspecto importante como antecedente de este programa son las consideraciones del Plan de Desarrollo (nacional, estatal o municipal). En relación con el Plan Nacional de Desarrollo PND, 2007 - 2012, las acciones de la SEDESOL buscan materializar el desarrollo humano sustentable. Así, en el PND se establecen los objetivos nacionales, estrategias y prioridades que busca el gobierno como base para los programas sectoriales, especiales, institucionales y regionales (SEDESOL, 2012).

\section{REVISIÓN TEÓRICA}

El programa Hábitat involucra varios conceptos claves relacionados con un mejoramiento de las condiciones de vida de la sociedad que habita en zonas urbanas con más de 15,000 habitantes. En este apartado se detalla el concepto de pobreza, marginación y política social que enmarcan a la sociedad que toca estudiar.

Sobre política social se puede definir de acuerdo con varios matices, pero Navarro (2011) lo muestra como un conjunto de estrategias concretas que aplica el Estado para que la sociedad viva de manera más equitativa. Esto tiene como propósito principal facilitar la convergencia entre los intereses individuales y comunales de los grupos humanos.

La política social también puede distinguirse desde dos perspectivas; desde un programa de gobierno y la acción del mismo. En relación con la primera perspectiva, los programas procuran llevar bienestar social por medio de políticas que los avalan y, por ese motivo, se ejercen medidas 
para tratar de mejorar la situación que se quiere aminorar. Las políticas son, en este sentido, momentáneas y surgen porque existen fallas en las políticas económicas que están en vigor. La segunda perspectiva considera que es necesario reducir o eliminar las desigualdades sociales y redistribuir recursos monetarios, proporcionar servicios asistenciales, crear oportunidades y desarrollar capacidades (asistencialismo) en rubros como salud, educación, seguridad social, vivienda, etc.

Se puede resumir el concepto de política pública como las decisiones que toma el gobierno para conservar y mejorar el bienestar social, procurando que alcancen a toda la sociedad los beneficios logrados con equidad y vida digna. Así, surge el concepto de Estado de Bienestar que se define como: "el conjunto de instituciones de regulación, redistribución de recursos e integración política(...)" (Navarro, 2011).

Además, el mismo autor señala que el Estado de Bienestar tiene diversas funciones ya sean económicas (corregir las fallas de mercado y equilibrar los ciclos económicos), sociales (redistribución del ingreso y reducción de desigualdades) o políticas (estabilidad social y orden político).

Conde, Ocaña y Pérez (2007) señalan que el tema del Estado de Bienestar está ganando espacios, sosteniéndose en el concepto de globalización y sostenibilidad financiera. Aunque hay otras corrientes que indican que, debido al envejecimiento de la población, será insostenible el Estado de Bienestar en relación con las pensiones.

El concepto anterior de Estado de Bienestar cae dentro del utilitarismo, por eso han surgido otras corrientes que muestran que hay una gran variedad de motivaciones para intentar lograr el bienestar, y no sólo maximizar o minimizar una variable. En este sentido, Amartya Sen sostiene que más allá del bienestar hay metas, y de las metas hay valores. Es decir, no todo lleva a cuestiones económicas (Gamboa y Cortez, 1999).

Los autores mencionados también afirman que las críticas para el utilitarismo pueden integrarse en dos puntos, en relación con que la utilidad es la única fuente de valor. El primero es que la utilidad es el reflejo del bienestar de una persona (pero el éxito de tal individuo no se puede evaluar en términos de su bienestar) y el segundo es que se puede discutir que el bienestar personal deba considerarse en términos de su utilidad, en lugar de otros términos. Por lo tanto, en cada persona coexisten las facetas 
de ser y de bienestar, aunque relacionadas, no son lo mismo. Además, las personas cambian continuamente sus gustos y preferencias, por eso cada individuo puede tener diversas razones para tener objetivos para su bienestar personal.

Entre los modelos que formalizan el bienestar social, Ruiz-Castillo (1998) muestra cómo estos cumplen con criterios de eficiencia y equidad. Indica que la Función de Bienestar Social (FBS) es una función real formando el espacio $(\mathrm{RH})$ de los ingresos personales, donde cada distribución del ingreso $\mathrm{x}=\left(\mathrm{x}_{1}, \mathrm{x}_{2} \ldots \mathrm{x}_{\mathrm{H}}\right)$ proporciona bienestar social.

Otro trabajo que formaliza esta teoría es el de Accinelli, et al. (2008). Ellos consideran una economía con " $n$ " agentes y "l” bienes. El consumo de cada agente se denota como $R_{+}^{l}$. Los agentes se representan como $\mathrm{I}=$ $(1,2, \ldots . \mathrm{n})$ y tienen utilidades $u_{i}$. Una asignación $\mathrm{x}=\left(\mathrm{x}_{1}, \mathrm{x}_{2}, \ldots \mathrm{x}_{\mathrm{n}}\right) \in \mathrm{R}^{\ln }$ es factible si y solo si $\sum_{\mathrm{i}=1}^{\mathrm{n}} \mathrm{x}_{\mathrm{i}}=\sum_{\mathrm{i}=1}^{\mathrm{n}} \mathrm{W}_{\mathrm{i}}$. Se representa por $\mathrm{F}$ el conjunto de asignaciones factibles. De este modo, la función de bienestar social de la economía se denota como: $\mathrm{W}_{\lambda}(\mathrm{x})=\sum_{i=1}^{n} \lambda_{\mathrm{i}} \mathrm{u}_{\mathrm{i}}\left(\mathrm{x}_{\mathrm{i}}\right)$.

Por lo que $\mathrm{x} \in \mathrm{R}^{\ln }$ es una asignación de recursos factibles, $\mathrm{y} \lambda=\left(\lambda_{1}\right.$, $\left.\lambda_{2} \ldots \lambda_{\mathrm{n}}\right)$ con $\lambda_{\mathrm{i}} \geq 0$ donde $\mathrm{i}=1, \ldots \mathrm{n}$, es el vector de las ponderaciones sociales de $\mathrm{R}^{\mathrm{n}}$.

Otro aspecto relacionado al bienestar social es la pobreza que afecta a las familias con bajos ingresos a nivel hogar. Díaz y Turner (2012) presentan una definición y criterios de medición de pobreza y definen el concepto como la carencia que tienen las personas para poder satisfacer sus necesidades mínimas como alimentación; por tanto, no aseguran su subsistencia. Esto implica que su medición se basa en términos del ingreso neto percibido y se establece una línea de pobreza que se compara con la canasta de bienes básicos que satisfacen el nivel mínimo de bienestar. De acuerdo con esto, surgen dos formas de medirlo, el enfoque directo (línea de pobreza) y el indirecto (necesidades básicas insatisfechas).

De acuerdo con el enfoque directo, las líneas de pobreza pueden clasificar a los hogares (población) en extrema y moderada pobreza, el primer caso presenta una negación o limitación de lo más mínimo para tener una vida con dignidad, por lo que puede caer en exclusión. De modo que un apoyo alimenticio no es suficiente para salir de esta condición, además que puede depender de los recursos disponibles en su 
medio ambiente. La pobreza moderada, por su parte, es otra línea de pobreza que está por arriba de la anterior pero que no implica un acceso libre a la salud y educación. Así, los hogares en extrema pobreza muestran una condición absoluta mientras que los hogares moderados una condición relativa.

Más formalmente, sea $\left(\mathrm{y}_{1}, \mathrm{y}_{2}, \ldots \mathrm{y}_{\mathrm{n}}\right)$ el vector de ingresos de la población (hogares) conformado por " $n$ " individuos, la función de pobreza $\pi$ $\left(y_{1}, z\right)$ indica que tan pobre es el individuo con su ingreso neto $\left(\mathrm{y}_{1}\right)$ cuando la línea de pobreza es " $z$ "; la cual se puede denotar como $H=\frac{1}{n} \sum_{i=1}^{n} 1\left(y_{1}\right.$ $<z)$, por lo que $\pi\left(y_{1}, z\right)=1\left(y_{1}<z\right)$. Así, se llega a una función binaria que si es igual a 1 el individuo (hogar) es pobre, y si esa función es igual a 0 entonces no es pobre (Chávez, et al. 2009).

En relación con el programa Hábitat y pobreza, Díaz y Turner (2012) elaboran un estudio para ciudades fronterizas del norte de México, donde muestran el comportamiento de la pobreza y el grado de marginación durante 2002, 2003 y 2004. Los resultados indican que en lo social no debe darse como ganada la lucha con estos problemas de la sociedad pues se acrecienta la cantidad de pobres debido a la llegada de inmigrantes. Además, la pobreza moderada está muy difundida y las condiciones de vida, el hábitat y la infraestructura han sido rebasadas por el crecimiento de la población y escasez de agua. Por ese motivo se sugiere que el gasto social ejercido por programas de apoyo a la sociedad debe ampliarse para fomentar la creación de las capacidades de la población, y con ello evitar que siga aumentando la pobreza extrema.

Así, la política social se rige por siete principios rectores: focalizar recursos en los programas más efectivos, instrumentar sistemas de evaluación y seguimiento que posibiliten la mejora continua, promover la coordinación de esfuerzos entre dependencias y organismos de los distintos órdenes de gobierno, priorizar acciones encaminadas a elevar las capacidades de las personas, utilizar un padrón único, vinculación adecuada entre programas y transparencia en la asignación y gasto de los recursos. 


\section{ZONA DE ESTUDIO}

En esta sección se caracterizan a los dos municipios sobre aspectos generales, demográficos y socio-económicos. Los municipios de Umán y Kanasín se localizan en colindancia con el municipio de Mérida y forman parte de la denominada zona metropolitana, que ha dado lugar a una zona urbana policéntrica (figura 1).

Figura 1

Localización de los municipios de Umán y Kanasín, Yucatán

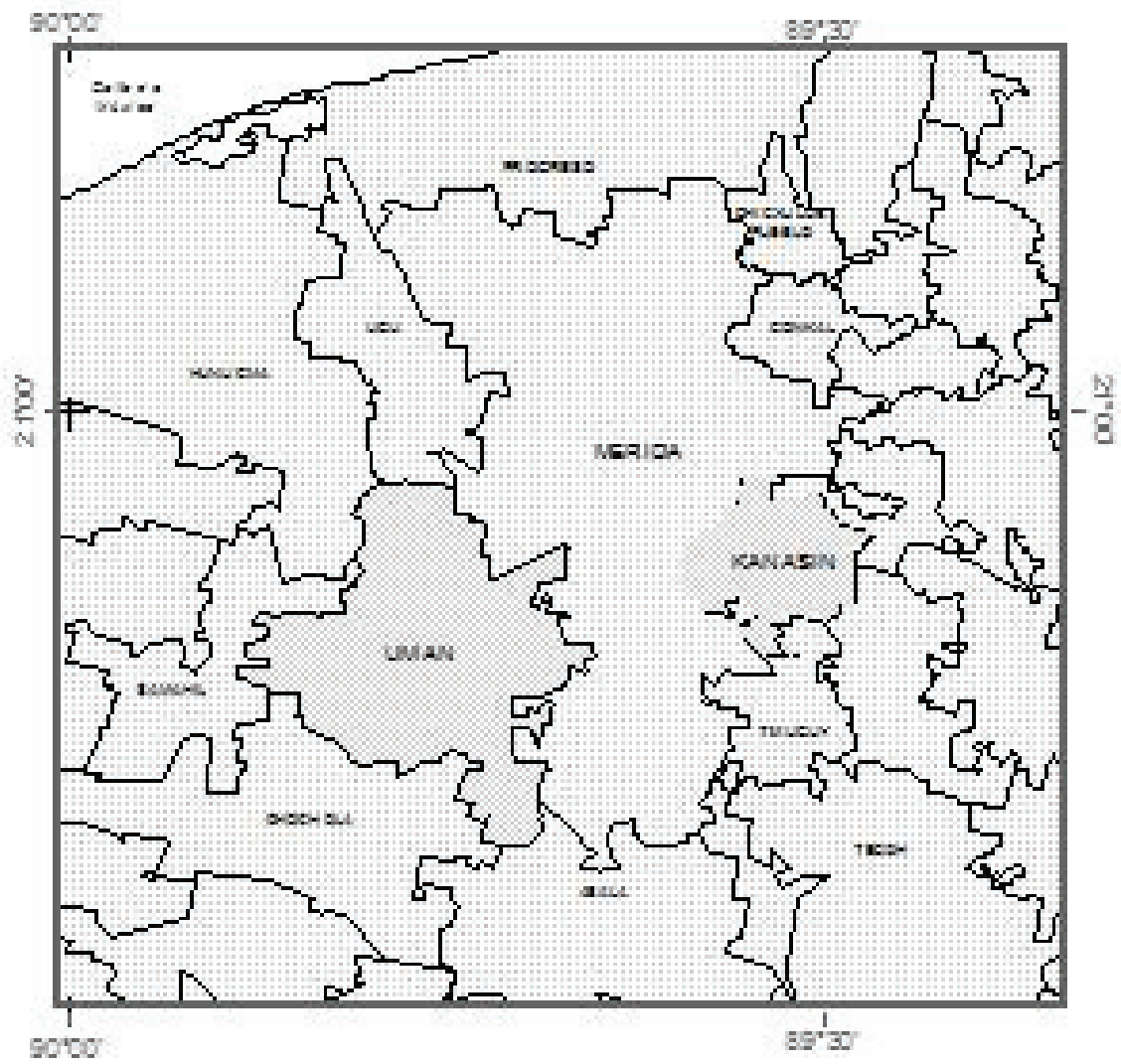

ruente: Elaboracion propia. 
a) Población. El municipio de Kanasín presenta un grado de urbanización de $96.1 \%$, valor alto que lo clasifica como municipio urbano en el contexto estatal. Está constituido por 29 colonias urbanas y 3 comisarías; ha crecido aceleradamente en los últimos 10 años, con un incremento de $100 \%$ en su población. En contraste, Umán mantiene un rápido crecimiento natural que se concentra en la cabecera municipal y tiene, aproximadamente, un grado de urbanización de 60 por ciento. Durante los 10 últimos años no muestra un crecimiento significativo de la población, a excepción de la zona conurbada con el municipio de Mérida (cuadro 4).

\section{Cuadro 4}

Variación poblacional (2000-2010)

\begin{tabular}{|l|r|r|r|}
\hline \multicolumn{1}{|c|}{ Municipio } & Población 2000 & Población 2010 & \multicolumn{1}{c|}{ Variación \% } \\
\hline Kanasín & 39,191 & 78,709 & 100.83 \\
\hline Umán & 49,145 & 50,993 & 3.76 \\
\hline Yucatán & $1,658,210$ & $1,955,577$ & 17.93 \\
\hline
\end{tabular}

Fuente: INEGI, 2000, 2010.

Los datos muestran que la población de Kanasín ha tenido un crecimiento más acelerado con respecto al estatal, ya que mientras la población de Yucatán creció tres veces de 1960 a 2005, la población de Kanasín creció diez veces en el mismo periodo. En el caso del municipio de Umán sólo se muestra un crecimiento de 0.6 veces durante el mismo periodo (POETY, 2006). Lo anterior se puede visualizar al mostrar la densidad poblacional de ambos municipios: Kanasín en el año 2000 tuvo 404 habitantes $/ \mathrm{km}^{2}$ y para 2010 fue de 534 habitantes $/ \mathrm{km}^{2}$, Umán pasó de 115 a 144 habitantes/ $\mathrm{km}^{2}$ durante el mismo periodo.

b) Población bilingüe. Con datos del Instituto Nacional de Estadística, Geografía e Informática, INEGI, es posible afirmar una tendencia al decrecimiento de la población bilingüe en la zona de estudio, destaca un importante decremento en Kanasín de casi $10 \%$ en una década, mientras que la de Umán es menor la pérdida (cuadro 5). 


\section{Cuadro 5}

Porcentaje de población mayor a 5 años, bilingüe

\begin{tabular}{|l|c|c|}
\hline \multicolumn{1}{|c|}{ Municipio } & \% Población bilingüe 2000 & \% Población bilingüe 2010 \\
\hline Kanasín & 28.7 & 19.7 \\
\hline Umán & 25.7 & 23.5 \\
\hline Mérida & 14.6 & 9.8 \\
\hline Yucatán & $\mathbf{3 7 . 3}$ & $\mathbf{3 0 . 3}$ \\
\hline México & $\mathbf{7 . 1}$ & $\mathbf{1 . 8}$ \\
\hline
\end{tabular}

Fuente: Calculado a partir de datos del INEGI, 2000, 2010.

c) Grado de marginación. En los años 2000 y 2010 Yucatán ocupó el $11^{\circ}$ lugar nacional como zona con alto grado de marginación y $77.4 \%$ de los municipios presentaban un rango de marginación alto y muy alto. En el otro extremo sólo tres municipios (Mérida, Progreso y Kanasín) se ubicaban en la franja de marginación baja y muy baja. Respecto de Umán, el crecimiento urbano se ha concentrado en la cabecera del municipio y en localidades cercanas a la cabecera. En el año 2000 fue clasificado con un grado de marginación medio, donde habitaba $18.56 \%$ del total de la población estatal. El cuadro 6 presenta un comparativo del índice de marginación, grado de marginación y el lugar que ocupan en el contexto nacional.

\section{Cuadro 6}

Comparativa de índices de marginación, 2000 y 2010

\begin{tabular}{|l|r|c|c|c|c|c|}
\hline $\begin{array}{c}\text { Estado/ } \\
\text { Municipio }\end{array}$ & $\begin{array}{c}\text { Población } \\
\text { total }\end{array}$ & $\begin{array}{c}\text { Índice de } \\
\text { marginación }\end{array}$ & $\begin{array}{c}\text { Grado } \\
\text { de marginación } \\
2000\end{array}$ & $\begin{array}{c}\text { Grado } \\
\text { de marginación } \\
2010\end{array}$ & $\begin{array}{c}\text { Lugar } \\
\text { nacional } \\
\text { que ocupa } \\
2000\end{array}$ & $\begin{array}{c}\text { Lugar } \\
\text { nacional } \\
\text { que ocupa } \\
2010\end{array}$ \\
\hline Kanasín & 78,709 & -1.0611 & Medio & Bajo & 1.73 & 2,08 \\
\hline Umán & 49,145 & -0.3318 & Medio & Bajo & 1.72 & 1,85 \\
\hline Yucatán & $1,658,210$ & 0.3813 & Alto & Alto & 11 & 11 \\
\hline
\end{tabular}

Fuente: Índices de marginación, Consejo Nacional de Población, Conapo, 2001, 2012, Plan de Desarrollo Urbano de Umán, 2001 - 2004. 
d) Características de la vivienda. La disponibilidad de servicios básicos (agua, drenaje y electricidad) son indicadores que inciden en el grado de bienestar dentro de la vivienda y, por tanto, influyen en la calidad de vida de sus ocupantes. Acorde con el incremento poblacional, la propagación de viviendas es marcadamente acelerada para Kanasín, con un aumento de $146 \%$ en la edificación en los últimos diez años, lo que influye directamente en la expansión de la mancha urbana y una demanda creciente de servicios; la cobertura en el municipio exhibe rezagos, principalmente en disponibilidad de drenaje, agua entubada y, en proporción menor, energía eléctrica (cuadro 7).

\section{Cuadro 7}

Características de la vivienda en Yucatán y Kanasín, 2000 y 2010

\begin{tabular}{|l|c|c|c|c|}
\hline $\begin{array}{l}\text { Características de la } \\
\text { vivienda }\end{array}$ & Kanasín 2000 & Kanasín 2010 & Umán 2000 & Umán 2010 \\
\hline $\begin{array}{l}\text { Total de viviendas } \\
\text { particulares habitadas }\end{array}$ & 8,363 & 20,309 & 10,506 & 12,748 \\
\hline Con agua entubada & 7,040 & 18,690 & 9,209 & 11,146 \\
\hline Con drenaje & 4,654 & 17,547 & 5,568 & 9,535 \\
\hline Con energía eléctrica & 7,986 & 19,607 & 10,204 & 12,564 \\
\hline
\end{tabular}

Fuente: INEGI, Censo general de población y vivienda, 2000, 2010.

En relación con Umán la cobertura de servicios de agua y energía eléctrica es amplia, pues de 18 localidades, más de $77 \%$ de las viviendas tiene los servicios. En energía eléctrica el panorama es más favorable, porque de esas mismas localidades más de $90 \%$ de viviendas cuentan con ella. El servicio de drenaje es inexistente, el municipio no dispone de infraestructura para el manejo de aguas residuales. Existe un serio problema de contaminación del suelo y del manto freático, fenómeno que se agrava con el crecimiento acelerado de la zona urbana de la ciudad de Mérida. 


\section{METODOLOGÍA}

Los resultados del presente trabajo corresponden a un diseño retrospectivo y descriptivo. Se trabajó en zonas de extrema pobreza correspondientes a Umán y Kanasín mediante un cuestionario estructurado, se obtuvo una muestra proporcional al número de familias existentes en los polígonos de las zonas de estudio, a la vez que la distribución de la misma fue homogénea con el criterio de igual cantidad de cuestionarios por manzana o cuadra. Se asignó un número consecutivo a las manzanas de los polígonos y se distribuyó la carga a los encuestadores.

Se tomó el criterio de aplicar de tres a cuatro cuestionarios en las zonas con mayor densidad poblacional, distribuidas equitativamente en la manzana, en los casos de cuadras con baja densidad poblacional se tomó el criterio de levantar la cantidad que la zona permitiera, que en su mayoría fue de tres hogares por manzana. Cabe mencionar que en el recorrido fue evidente la escasa presencia de beneficiarios del programa Hábitat, por lo que se tomó en consideración la importancia de captar en campo a los beneficiarios e incorporarlos en la encuesta.

Para ello se diseñó un cuestionario de carácter multidimensional que abarca aspectos de actores sociales (involucrados en la operación e impacto del programa), distintas áreas de conocimiento (física y perceptual), temáticas que se trabajan (habitabilidad, bienestar en los hogares, participación social, desarrollo comunitario, accesibilidad laboral) y temas específicos tales como: imagen urbana, pobreza, equidad, relaciones familiares, acceso a una vida digna, oportunidades de aprendizaje, empleo mejor remunerado, participación social, etc.

A través de la indagación sistemática se recogió información documental de las áreas beneficiadas con el programa, así como las mejoras registradas. Después se realizó una investigación documental cuyo objetivo fue recabar información necesaria para conocer la percepción de la población sobre el programa, así como identificar a los informantes clave; aunque para el caso de Kanasín no fue posible contar con registros y tampoco contactar con los responsables del ejercicio al que el estudio compete (2005 - 2006). Asimismo, la identificación de informantes para la realización del trabajo de investigación con beneficiarios presentó dificultades 
importantes como, por ejemplo, no contar con listados de los beneficiarios 2005 - 2006, de tal suerte que fue necesario detectarlos a través de la encuesta en campo, así como en los Centros comunitarios.

Más adelante se procedió a realizar entrevistas individuales y grupales a beneficiarios del programa Hábitat. El propósito fue la obtención del punto de vista de la población sobre los beneficiarios del programa (cursos, obras para mejoramiento de los barrios y grado de participación de los actores sociales). Se aplicaron encuestas a hogares en los polígonos Hábitat para obtener información sobre el ingreso y gasto.

Se hicieron 675 encuestas a hogares en cuatro polígonos considerados por el programa Hábitat de Kanasín que representaron 2,804 personas de las familias; mientras que en Umán se realizaron 459 entrevistas a hogares que vinculan a 1,976 personas. El cuestionario aplicado en la encuesta es de tipo estructurado, cerrado y sometido a pilotaje.

Para el análisis de datos se construyeron indicadores tomando en cuenta los ejes temáticos que aborda el programa Hábitat, lo que permitió caracterizar los resultados y otorgar valores cuantificables para las acciones Hábitat. De tal forma que se desarrollaron dos tipos de indicadores: de magnitud (inversión per cápita y cobertura) y de impacto o efecto (remuneración salarial, pobreza patrimonial, servicios básicos en las viviendas, condiciones de vida, participación social, desarrollo comunitario, etc.). En algunos casos se comparan los resultados de la encuesta realizada con estadísticas del INEGI (2005).

\section{RESULTADOS Y ANÁLISIS DE INFORMACIÓN}

\subsection{Características socio-económicas de los polígonos Hábitat}

Según los resultados de las encuestas realizadas en Kanasín y Umán se tiene que, en el primer municipio, el estado civil de la mayoría de los encuestados es soltero, seguido por casados como los más numerosos. En relación con Umán, $47 \%$ se declaró soltero, $45 \%$ casado, $2.7 \%$ viudos o viudas, $2.4 \%$ se encontraban en unión libre, $1.6 \%$ separadas, $0.46 \%$ divorciadas y $0.61 \%$ madres solteras. 


\section{Cuadro 8}

Actividades realizadas por la población de Kanasín y Umán

\begin{tabular}{|l|c|c|c|c|}
\hline \multicolumn{1}{|c|}{ Actividad } & $\begin{array}{c}\text { Kanasin- } \\
\text { Frecuencia }\end{array}$ & $\%$ & $\begin{array}{c}\text { Umán- } \\
\text { Frecuencia }\end{array}$ & $\%$ \\
\hline Estudiantes (recién egresados) & 762 & 28.4 & 588 & 32.3 \\
\hline Empleados & 612 & 22.8 & 422 & 23.1 \\
\hline Amas de casa & 409 & 15.3 & 425 & 23.3 \\
\hline Trabajadores independientes & 261 & 9.7 & 184 & 10.1 \\
\hline Empleados de construcción & 75 & 2.8 & 37 & 2.0 \\
\hline Obreros & 70 & 2.6 & 98 & 5.4 \\
\hline Desempleados & 124 & 4.6 & $\mathrm{~s} / \mathrm{d}$ & - \\
\hline Otras actividades* & 266 & 13.7 & 17 & 0.9 \\
\hline Total & $\mathbf{2 5 7 9}$ & $\mathbf{1 0 0 . 0}$ & $\mathbf{1 ~ 8 2 4 * *}$ & $\mathbf{1 0 0 . 0} * *$ \\
\hline
\end{tabular}

Fuente: Elaborado con base en información generada por los autores.

*Incluye carniceros, soldadores, enfermeros, jubilados, transportistas.

**No coincide el total con la sumatoria porque hay 53 actividades realizadas que no se lograron identificar.

Entre las principales actividades realizadas por los beneficiarios del programa Hábitat están los estudiantes que concluyeron su educación primaria y secundaria (cuadro 8). También se obtuvo que, en promedio, 36\% de los trabajadores laboran más de 8 horas diarias, $61.6 \%$ trabaja entre 5 y 8 horas y el $2.2 \%$ trabaja entre 1 y 4 horas diarias.

De las personas que reciben ingreso por alguna actividad productiva, por salarios mínimos, en Kanasín corresponde a familias con jefatura masculina $86.8 \%$ y con jefatura femenina $11.4 \%$, aunque $1.8 \%$ de los hogares no fueron determinados. En Umán, predominan las familias con jefatura masculina, $91.1 \%$ y con jefatura femenina $8.5 \%$. La distribución de ingresos por salarios mínimos se desglosa en el cuadro 9. 


\section{Cuadro 9}

Ingresos diarios de la población de Kanasín y Umán

\begin{tabular}{|l|c|c|c|c|}
\hline Ingresos diario & $\begin{array}{c}\text { Frecuencia- } \\
\text { Kanasín }\end{array}$ & $\%$ & Frecuencia-Umán & $\%$ \\
\hline Cero pesos & 1,364 & 48.7 & 1,193 & 60.4 \\
\hline De \$1 a \$51.95 pesos & 406 & 14.5 & 36 & 1.8 \\
\hline De \$51.96 a \$103.05 & 492 & 17.6 & 422 & 21.4 \\
\hline De \$103.06 a \$155.85 & 296 & 10.6 & 223 & 11.3 \\
\hline De \$155.86 a \$207.08 & 108 & 3.9 & 65 & 3.3 \\
\hline De \$207.09 a \$259.75 & 29 & 1.0 & 14 & 0.7 \\
\hline Más de \$259.76 & 38 & 1.4 & 23 & 1.2 \\
\hline Total & $\mathbf{2 ~ 8 0 3}$ & $\mathbf{1 0 0 . 0}$ & $\mathbf{1 9 7 6}$ & $\mathbf{1 0 0 . 0}$ \\
\hline
\end{tabular}

Fuente: Elaborado con base en información generada por los autores.

En relación con las enfermedades más comunes, en Kanasín se encuentran las respiratorias, seguidas por las gastrointestinales $(52.4 \%$ y $9.2 \%$, respectivamente), a pesar de que tienen cobertura de salud $78.3 \%$ de la población. Entre tanto, en Umán se reporta el mismo comportamiento de enfermedades, con repunte de las respiratorias de 82.3 por ciento.

Respecto de los tipos de viviendas, estas varían considerando las condiciones de construcción. Entre los beneficiarios del programa Hábitat, en Kanasín y Umán se encuentra que la mayoría son urbanas, seguidas por la categoría en "transición" (cuadro 10). Los tipos de piso de las viviendas que están relacionadas con lo anterior, pueden ser de cemento y mosaico principalmente (53\% y $43 \%$ ) en ambos municipios. De igual modo, en promedio $60 \%$ de las viviendas cuentan con más de tres cuartos, seguidas de entre 2 y 3 cuartos (36\% en promedio en los dos municipios). Sólo $3.75 \%$ tiene un solo cuarto en las viviendas. 


\section{Cuadro 10}

Tipos de vivienda en Kanasín y Umán

\begin{tabular}{|l|c|c|c|c|}
\hline \multicolumn{1}{|c|}{ Tipos de vivienda } & Vivienda - Kanasín & $\%$ & Vivienda - Umán & $\%$ \\
\hline Urbana & 496 & 73.5 & 394 & 86.0 \\
\hline Transición & 129 & 19.1 & 51 & 11.0 \\
\hline Interés social & 42 & 6.2 & 9 & 1.96 \\
\hline Maya & 3 & 0.4 & 4 & 0.87 \\
\hline No identificados & 5 & 0.7 & 1 & 0.22 \\
\hline Total & $\mathbf{6 7 5}$ & $\mathbf{1 0 0}$ & $\mathbf{4 5 9}$ & $\mathbf{1 0 0}$ \\
\hline
\end{tabular}

Fuente: Encuestas de campo.

En los hogares se declaró que en su mayoría se usa gas como combustible (73\% para Kanasín y $56.2 \%$ para Umán), seguido del uso de leña o carbón ( $24 \%$ y $35.3 \%$, respectivamente).

\subsection{Percepción de los hogares al programa Hábitat (servicios públicos)}

Con base en la opinión de las personas, en los polígonos Hábitat se aprecia que se tiene una percepción diversa de los servicios públicos recibidos, sin embargo, en ambos municipios la pavimentación de calles y la mejora en el alumbrado público son las más destacadas (gráfica 1).

\section{Gráfica 1}

Cantidad de habitantes que perciben mejoras en servicios públicos

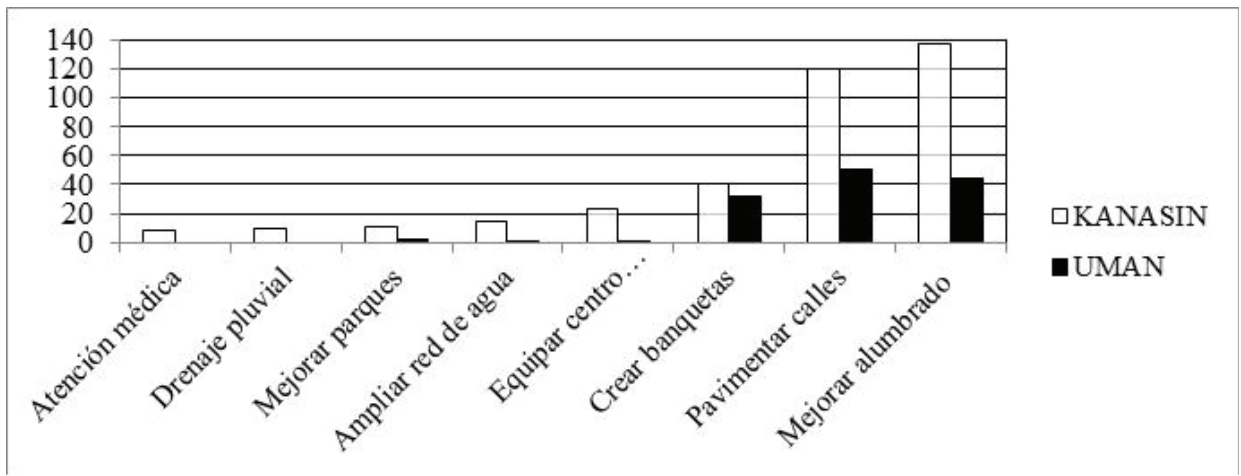

Fuente: Elaborada con base en información generada por los autores. 
En Kanasín, de 675 hogares, 70.7\% de los encuestados consideran que tienen todos los servicios públicos en sus calles, $2.2 \%$ opina lo contrario y $27.1 \%$ no pudo responder con certeza. En cuanto a Umán, los resultados muestran que de 459 hogares encuestados; 76.9\% consideró que su calle cuenta con todos los servicios públicos, $20.5 \%$ señaló que no tienen completos los servicios públicos y $2.6 \%$ no sabe o no externó opinión al respecto. Entre los cursos del programa Hábitat que se impartieron en Kanasín los entrevistados reconocieron muy pocos (cuadro 11).

\section{Cuadro 11}

Eventos organizados por el proyecto Hábitat (2005 - 2008)

\begin{tabular}{|l|c|c|}
\hline Curso & $\begin{array}{c}\text { Participantes } \\
\text { en Kanasín }\end{array}$ & $\begin{array}{c}\text { Participantes } \\
\text { en Umán }\end{array}$ \\
\hline Repostería & 16 & 7 \\
\hline Costura & 14 & 7 \\
\hline Cultura de belleza & 7 & 4 \\
\hline Corte y confección & 6 & 3 \\
\hline Manualidades & 4 & 5 \\
\hline Electricidad residencial & 3 & 9 \\
\hline Torneo de futbol & 2 & 0 \\
\hline Baile y danza & 1 & 3 \\
\hline Acondicionamiento físico & 1 & 0 \\
\hline Fortalecimiento de la familia & 1 & 0 \\
\hline Urdido de hamacas & 1 & 3 \\
\hline Otros (plomería, carpintería, etc.) & 0 & 8 \\
\hline Total & $\mathbf{5 6}$ & $\mathbf{5 0}$ \\
\hline
\end{tabular}

Fuente: Elaborado con base en información generada por los autores.

Sobre el número de cursos en los cuales han participado (gráfica 2) se observa irregularidad, ya que, a pesar que Kanasín es más populoso, Umán tiene un número de participantes similar a Kanasín. En ninguno de los dos municipios los participantes declararon involucrarse en cursos de otros años (mixtos). En cuanto a oferta-demanda de los cursos que otorga el programa, en los cuatro años registrados, la participación es mayor en 2006 y en 2008, destaca un mayor porcentaje en Kanasín. 


\section{Gráfica 2}

Número de participantes de los cursos por municipio (2005-2008)

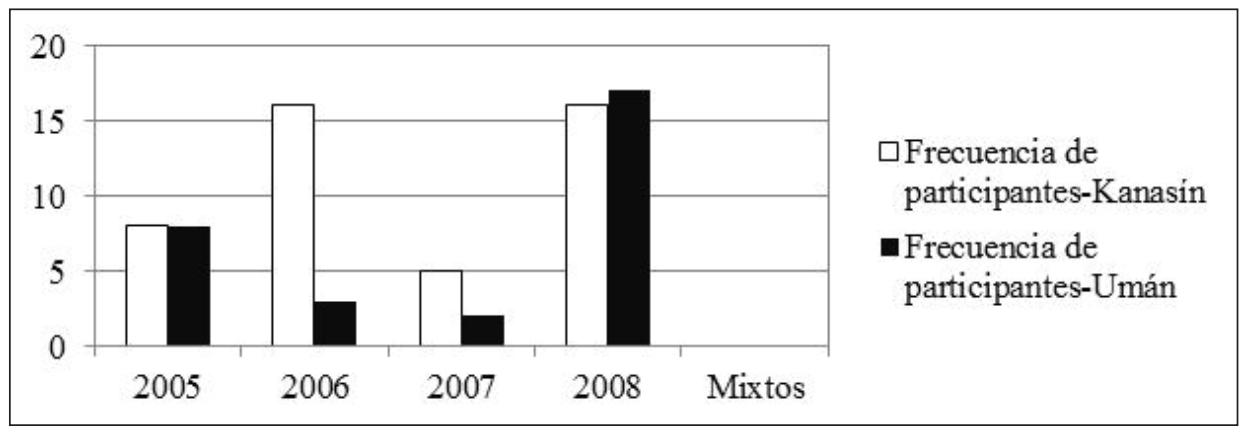

Fuente: Elaborada con base en información generada por los autores.

Se espera como una medida de éxito del programa Hábitat que los beneficiarios utilicen los conocimientos adquiridos para mejorar su salario y lo apliquen a sus negocios propios, no obstante no se vislumbra tal acción. También es posible apreciar que la familia en lo general no se apropia del aprendizaje adquirido por algún integrante, pues no lo replican. Así, los resultados de tal difusión y aplicación no son lo esperado, ya que en ninguno de los municipios se evidenció la participación de más de 20 familias, si se tiene en cuenta que fueron encuestados 675 hogares en Kanasín y 459 hogares en Umán (gráfica 3).

\section{Gráfica 3}

Difusión del conocimiento y habilidades del programa Hábitat

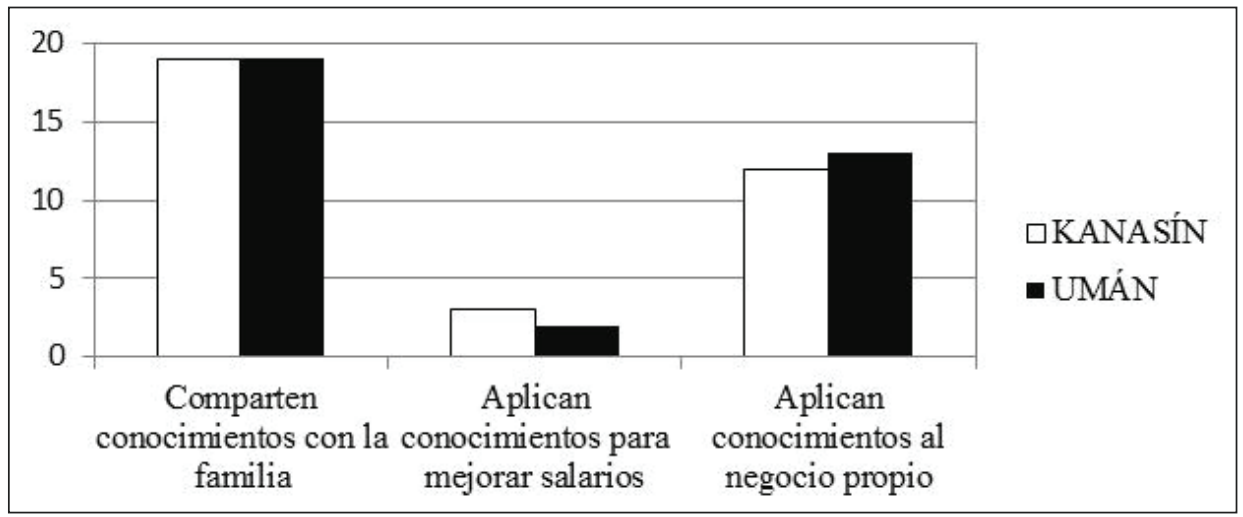

Fuente: Elaborada con base en información generada por los autores. 
A pesar de los resultados de la poca incidencia de los cursos del programa Hábitat, los reportes oficiales indican que el programa ha tenido una importancia creciente en los municipios estudiados. El cuadro 12 muestra tal comportamiento, se reporta que en Kanasín había 4,147 habitantes en los polígonos Hábitat y 3,016 en Umán.

\section{Cuadro 12}

Población atendida y porcentaje de cobertura Hábitat. Kanasín y Umán

\begin{tabular}{|l|c|c|c|c|}
\hline $\begin{array}{c}\text { Acciones } \\
\text { 2005-2006 }\end{array}$ & $\begin{array}{c}\text { Población } \\
\text { atendida Kanasin } \\
2005-2006\end{array}$ & $\begin{array}{c}\% \\
\text { de cobertura } \\
\text { Kanasin }\end{array}$ & $\begin{array}{c}\text { Población } \\
\text { Umán atendida } \\
2005-2006\end{array}$ & $\begin{array}{c}\% \\
\text { de cobertura } \\
\text { Umán }\end{array}$ \\
\hline Cursos & 1,369 & 33 & 1,551 & 51.42 \\
\hline Habilitación centros & 620 & 14.95 & 395 & 13.1 \\
\hline $\begin{array}{l}\text { Consultas y/o } \\
\text { campañas de salud }\end{array}$ & 4,000 & 96.45 & 3,500 & 116 \\
\hline Obra civil & 19,205 & 463.10 & 0 & 0 \\
\hline Total & $\mathbf{2 5 1 9 4}$ & -- & $\mathbf{5 4 4 6}$ & $\mathbf{1 8 0}$ \\
\hline
\end{tabular}

Fuente: Elaborado con base en información generada por los autores.

También se muestra que existe una aplicación desigual de las actividades realizadas dentro del programa, cuando se considera a la población en general y se observa una gran diferencia presupuestal del gasto del programa Hábitat en Kanasín con respecto a Umán (cuadro 13).

\section{Cuadro 13}

Inversión de acciones del programa Hábitat en Kanasín y Umán

\begin{tabular}{|l|c|c|c|c|}
\hline $\begin{array}{c}\text { Acciones } \\
\text { 2005-2006 }\end{array}$ & $\begin{array}{c}\text { Inversión 2005 } \\
\text { Kanasín } \\
\text { (en pesos } M X)\end{array}$ & $\begin{array}{c}\text { Inversión } \\
\text { per cápita } \\
\text { Kanasín }\end{array}$ & $\begin{array}{c}\text { Inversión 2005 } \\
\text { Umán } \\
\text { (en pesos } M X)\end{array}$ & $\begin{array}{c}\text { Inversión } \\
\text { per cápita } \\
\text { Umán }\end{array}$ \\
\hline Cursos & 1976026 & 1443 & 2359205 & 152108 \\
\hline Habilitación centros & 2958192 & 4771 & 1348948 & 341505 \\
\hline $\begin{array}{l}\text { Consultas y/o } \\
\text { campañas de salud }\end{array}$ & 158000 & 39.5 & 319410 & 91.26 \\
\hline Obra civil & 32462936 & 1690.3 & 0 & 0 \\
\hline Total & $\mathbf{3 7 5 5 5 1 5 4}$ & $\mathbf{1 4 9 0 . 1}$ & $\mathbf{3 7 0 8} \mathbf{1 5 3}$ & $\mathbf{6 8 0 8 9 4}$ \\
\hline
\end{tabular}

Fuente: Elaborado con base en información generada por los autores. 


\subsection{Particularidades de los polígonos Hábitat Kanasín}

Entre las particularidades detectadas en Kanasín se pueden mencionar: escasez de espacios públicos destinados a recreación y deporte, falta de limpieza de predios y calles, calles sin pavimentar, viviendas contrastantes de la zona centro, tamaño de la vivienda y del predio, centros comunitarios reducidos por la existencia de una zona habitacional con problemas de violencia y falta de seguridad pública.

Por su parte, se registran dificultades en la operatividad del programa, y se encontró que no existe correspondencia entre el inicio de los cursos y la asignación del material necesario, dicho desfase obedece a las diferencias en tiempos de entrega de los recursos federales y estatales, en los que éste último debe aportar primero para acceder al recurso federal. Es así que hay un retraso que lleva a los usuarios a organizarse para cooperar y comprar los materiales indispensables para no suspender los cursos.

Asimismo, se registró la falta de difusión del programa entre la comunidad, pues como resultado de la encuesta realizada en los centros comunitarios se obtuvo que: 14 personas $(16.3 \%)$ afirman conocer el programa, $51(59.3 \%)$ no lo conocen, $7(8.1 \%)$ indican que les sirve para aprender algo constructivo, 2 (2.3\%) piensan que beneficia a las personas, $4(4.7 \%)$ consideran que sirve para ayudar y enseñar a personas de bajos recursos y 8 personas $(9.3 \%)$ opinan que es un beneficio para la familia.

En cuanto a la difusión de los cursos: 37 personas (43\%) afirman que se enteraron del programa por medio de anuncios, $29(33.7 \%)$ a través de una persona conocida, $10(11.6 \%)$ pasaron a invitarlos a participar, 7 $(8.1 \%)$ por un voceador, $2(2.3 \%)$ por la maestra y 1 persona $(1.2 \%)$ se enteró en una campaña política.

Así también se registró que las instalaciones de los centros comunitarios presentan deficiencias en cuanto a espacio adecuado, ventilación y equipo necesario, información que fue recabada de las entrevistas a 86 usuarias, tal y como se aprecia a continuación:

Respecto de la pregunta sobre si el programa cumple o no con las expectativas de la población, la mayoría (19 personas o $22.1 \%$ ) se abstuvo de responder, $6(6.9 \%)$ consideran que necesitan que se les proporcione material, 7 (8.1\%) que se impartan más cursos, 19 (22.1\%) centran sus 
necesidades en salud e higiene, $12(13.9 \%)$ consideran la falta cursos de cultura de belleza, $2(2.3 \%)$ solicitan internet y 3 personas $(3.5 \%)$ consideran que hacen falta actividades para niños.

Los polígonos Hábitat de Kanasín, según información de SEDESOL (2005), estaban conformados por más de 60\% de hogares en situación de pobreza extrema. El análisis general de esta zona arroja que $80 \%$ recibe hasta dos salarios mínimos como ingreso, porcentaje que indica una brecha salarial por encima a la registrada en el año 2000 (INEGI, 2000) de $70.3 \%$. Este dato es distinto a nivel hogar, ya que $27.8 \%$ percibe hasta dos salarios mínimos, valor que debe ser comparado con el promedio de los integrantes por familia (ingreso per cápita), que, según la encuesta realizada, es de 44 pesos (año 2005, zona C), cantidad que los ubica en el rubro de pobreza patrimonial.

El indicador relevante en el análisis de la pobreza en Kanasín es la relación ingreso-gasto; pues $35.5 \%$ de las familias destinan una cantidad importante de sus ingresos en alimentación. En un nivel medio de gasto, $35.9 \%$ de familias obtienen otros satisfactores y sólo $29.6 \%$ de los hogares registran una relación ingreso-gasto adecuada para cubrir otras necesidades. En síntesis, 70\% de las familias presenta pobreza patrimonial y el Consejo Nacional de Evaluación de la Política de Desarrollo Social, Coneval (2005) registra 69.7\% de este tipo de pobreza en Kanasín. Es posible concluir que el programa Hábitat no refleja un impacto directo en la disminución de la pobreza de las zonas de atención prioritaria.

Otro indicador de pobreza patrimonial se refiere a que para el año 2008 el valor de esta canasta fue de $\$ 200$ pesos y que $33.5 \%$ de las familias tienen un ingreso menor a dicha cantidad; lo que muestra a los hogares en pobreza alimentaria y patrimonial, y si a este porcentaje se le suma $48.6 \%$ que no recibe ingresos, entonces se obtiene un porcentaje mayor a 80 por ciento.

\subsection{Particularidades de los polígonos Hábitat Umán}

Los polígonos de Umán mostraron un desequilibrio en la distribución de la densidad poblacional y en la arquitectura urbana, ya que las dimensiones de los predios no son homogéneos, sólo el polígono conocido 
como " 050 " aglutina a la mayor cantidad de habitantes, por lo que se aprecia una zona de alta densidad poblacional, predios cada vez más pequeños y escasos terrenos baldíos. Concentra zonas de servicios y a la vez es el más urbanizado.

Por otra parte, se encuentran los polígonos de más reciente crecimiento, se observó que en ellos predomina una baja densidad poblacional, con diferencias en inversión en equipamiento urbano, ya que hay contrastes entre polígonos con importante porcentaje de calles pavimentadas y banquetas. Se encontraron zonas con actividad del programa Hábitat para pavimentación y drenaje urbano, aunque $90 \%$ de la población tiene cubiertos los servicios de luz eléctrica y agua potable.

El tipo de vivienda predominante en las colonias es urbano, con materiales durables; sin embargo, se identificaron zonas de viviendas edificadas con materiales frágiles. En los alrededores de las viviendas se encuentran solares con cultivos de árboles frutales y cría de animales, sobre todo en las zonas cuyos predios son de mayor tamaño, correspondientes a las zonas no céntricas.

En cuanto a la salud ambiental fue posible advertir que se encontraban en buenas condiciones, pero en los polígonos más lejanos el acceso es más difícil por la ausencia de calles pavimentadas y presencia de lotes baldíos, lo cual genera proliferación de fauna nociva; aunque por lo general las calles cuentan con alumbrado público.

\subsubsection{Descripción del polígono 050-Umán}

Las personas lo nombran indistintamente como San Lázaro y San Francisco. Entre las características del polígono se encuentra que está altamente poblada en alrededor de $90 \%$, no cuentan con servicio telefónico domiciliar, los lotes tienen un alto valor comercial y son escasos los baldíos, se encuentran obras o casas de interés social, no tienen la infraestructura urbana básica (pavimentación, alumbrado, etc.).

También se encontró que hay dos centros comunitarios, existen granjas en decadencia y estos terrenos en perspectiva serán para uso habitacional. Se observó que existen pocas casas con techos de lámina o con techos de materiales frágiles. 


\subsubsection{Observaciones sobre otros polígonos de Umán}

El mismo resultado de Kanasín se observa también en Umán respecto de la operatividad del programa, pues no existe correspondencia entre el inicio de los cursos y la asignación del material necesario para la práctica de los mismos. Asimismo, se registró la falta de difusión del programa entre la comunidad, como resultado de la encuesta realizada en los centros comunitarios 38 personas $(43.2 \%$ ) conocen el programa, mientras que 50 personas (56.8\%) no lo conocen, de modo que no existe claridad en la mayoría de la población sobre los beneficios del programa.

Sobre los canales de divulgación del programa Hábitat, 26 personas (29.6\%) se enteraron por medio de anuncios, 18 (20.5\%) fue porque lo pasaron a invitar; $12(13.6 \%)$ se enteraron por invitación de una vecina y 10 personas $(11.4 \%)$ por invitación de algún conocido.

Así también se registraron las necesidades y expectativas, información recabada en los centros comunitarios, y cuyos resultados son que: sólo 55 personas (62.5\%) han utilizado los servicios de clases; 14 (15.9\%) respondieron que no han utilizado otros servicios y 10 personas $(11.4 \%)$ no respondieron a esta pregunta.

Las instalaciones de los centros comunitarios se encuentran en buen estado y con aire acondicionado, destaca la infraestructura para los cursos de cultora de belleza. Es importante mencionar que las autoridades decidieron especializar los centros comunitarios, de esta forma el equipamiento corresponde al tipo de cursos que se programó desarrollar. Las usuarias manifestaron inconvenientes en relación con la oportunidad de acceder a los de vecindarios distintos y señalan que el centro comunitario San Francisco está masculinizado por este tipo de cursos y se requieren más horarios.

Sobre el impacto en la remuneración salarial en Umán, si bien no se cuenta con datos específicos para la zona de estudio, la encuesta realizada en los polígonos Hábitat indica que $60.4 \%$ no recibe ingresos y $21.4 \%$ de la población ocupada recibe hasta dos salarios mínimos, que al sumarlos significan $81.8 \%$, dato que apunta a una tasa de dependencia económica muy alta. 
Desde el punto de vista de los usuarios, en el salario o en el empleo 72 personas $(81.8 \%)$ consideran que el programa ayuda a mejorar las oportunidades en el empleo y 8 personas $(9.1 \%)$ no saben.

La situación de pobreza en los polígonos Hábitat de Umán según SEDESOL (2005) estaba conformado por más de $60 \%$ de hogares en situación de pobreza extrema. El análisis general de esta zona arroja que $81.8 \%$ recibe hasta dos salarios mínimos como ingreso, porcentaje que indica una brecha salarial por encima a la registrada en el año 2000 de 71 por ciento. Este dato es distinto a nivel hogar, ya que $21.4 \%$ percibe hasta dos salarios mínimos, valor que debe ser analizado a la luz del número de integrantes en promedio por familia, es decir, el ingreso per cápita, que correspondía a 48 pesos, cantidad que se ubica en el rubro de pobreza patrimonial.

Existe otro indicador de pobreza patrimonial y es el referido a contar con un ingreso necesario para comprar la canasta básica alimentaria. Para 2008 se estimó en 200 pesos el costo de dicha canasta y 56.5\% de las familias obtienen un ingreso menor a dicha cantidad, por lo que esta proporción se ubica en pobreza alimentaria y patrimonial.

Otro indicador relevante en el análisis de la pobreza es la relación ingreso gasto. Las familias destinan hasta $60 \%$ de su ingreso y se les clasifica en situación de pobreza patrimonial, al no contar con recursos para la totalidad de satisfactores básicos. Este análisis nos indica que $53.5 \%$ de las familias destinan una cantidad importante de sus ingresos en alimentación y únicamente $23 \%$ de los hogares registra una relación ingreso gasto adecuada para cubrir otras necesidades.

En relación con el punto de vista de las usuarias sobre el programa y la disminución de la pobreza, 36 personas (40.9\%) contestaron que si ayuda a dicho propósito, 23 (26.1\%) consideran que depende de la persona, $17(19.3 \%)$ opinan que en algunos casos si ayuda y 8 personas $(9.1 \%)$ no respondieron esa pregunta.

En cuanto a la percepción que el programa ejerce sobre el salario o empleo; 72 personas $(81.8 \%)$ consideran que el programa Hábitat ayuda a mejorar las oportunidades en el empleo o el salario, mientras que $9.1 \%$ no saben. Sobre quién es el más beneficiado del programa, si se considera el género de la población estudiada, los resultados muestran que 31.8\% piensa que son las mujeres. 


\subsection{Habitabilidad de barrios en polígonos en pobreza extrema (Kanasín y Umán)}

Kanasín: de acuerdo con los resultados únicamente 1.6\% de los encuestados manifiestan que hay condiciones precarias de vida por: falta de pavimentación, insuficiente alumbrado, falta de vigilancia y violencia en las calles, entre otros problemas; $9.5 \%$ expresa tener una vida satisfactoria.

El punto de vista que se obtiene de las usuarias sobre el programa a nivel colonia arroja que $87.2 \%$ de ellas considera que si hay un impacto, $5.8 \%$ indicó que no lo hay, mientras que el resto no sabe o no contestó. Este resultado permite observar que, en términos generales, existe una buena percepción del programa, aunque la población desconoce el funcionamiento y los cursos ofrecidos.

Umán: Únicamente $1.6 \%$ de los hogares y las colonias manifiestan condiciones de vida precarias, principalmente por: falta de pavimentación, alumbrado insuficiente, falta de vigilancia, violencia en las calles, entre otros problemas; $9.5 \%$ de las colonias sugieren tener una vida satisfactoria al encontrarse en el rango aceptable.

Es necesario incluir en este análisis el comparativo de las características de las viviendas entre las del año 2005 y 2010, recogidas en las encuestas a los polígonos Hábitat. En el 2005 1.9\% de las viviendas tenían piso de tierra y en 2010 solamente $0.7 \%$, lo que indica un cambio sustancial.

En cuanto a paredes de viviendas, $4.6 \%$ son de materiales precarios, para 2005 no se cuenta con el dato. De igual manera, se registró que 4.6\% de las viviendas tienen techo de materiales frágiles y para 2005 no se encuentra el dato.

Por lo que hace a servicios, en 2005: 4.4\% de las viviendas no contaba con agua potable en su domicilio, en la encuesta se encontró un ligero aumento a $4.5 \% ; 2.6 \%$ no contaba con energía eléctrica, en el estudio se registró $2.2 \%$, dato que revela un ligero descenso; $22.3 \%$ no tenía excusado y en la zona de estudio se registró $33.7 \%$, es decir, una tendencia considerable al aumento; principalmente se conectaba el drenaje a la fosa séptica. En resumen, es posible advertir mejoras tangibles en las características de las viviendas en esta localidad tras el proyecto Hábitat, básicamente en los pisos. 
Al realizar el análisis de habitabilidad con base en la ponderación de elementos descritos en la metodología, $40 \%$ de los hogares encuestados se encuentran en el nivel uno, nivel en el que se reconocen carencias en la infraestructura urbana y los servicios de las viviendas. Asimismo, destaca que en Umán no se registró un contraste entre sectores y sus condiciones de vida son homogéneas, pues el restante $60 \%$ de los hogares se ubica en un nivel medio de habitabilidad.

Las características de las viviendas de Umán resaltan al tener, en promedio, más de tres habitaciones y ser en su mayor parte de mampostería, situación que conlleva un bajo nivel de hacinamiento y vulnerabilidad al ambiente. También es de mencionar que, en todo momento del estudio, se encontraron maquinaria y materiales para obra civil en las colonias ubicadas en los polígonos Hábitat. Las calles son limpias y se observa poco alcoholismo en los barrios, como que la habitabilidad es buena en esta localidad y sus habitantes lo reconocen, pero demandan más servicios.

\subsection{Bienestar en los hogares}

Kanasín: el indicador de bienestar se construyó con los componentes ingresos, relación ingreso y gasto y, por último, la situación de salud. Los resultados arrojaron un mínimo de bienestar, pues se reportaron hogares con cero ingreso, que significó $3.5 \%$ de los hogares encuestados.

En este rubro se aprecia la capacidad económica para obtener una vida con satisfactores básicos y su repercusión en la salud de los integrantes de los hogares. No se registra análisis similar alguno en estudios o evaluaciones realizadas al programa Hábitat, por lo que no es posible establecer un comparativo. Aun así, se puede concluir que persiste en 20082009 un porcentaje de población que vive en condiciones de precariedad para satisfacer sus necesidades básicas, que el programa debe enfocarse a desarrollar estrategias que impacten directamente en el bienestar de las familias.

Así, la percepción de las participantes del programa señala que lo aprendido en los cursos es básico y que con ello no es posible iniciar un negocio propio, pues se requiere otro curso más para abundar. Además, 
señalan que existe un beneficio personal y familiar, ya que las mujeres se sienten más capaces y, al mismo tiempo, se ahorran un gasto al hacer ellas en casa lo aprendido. Se concluye que, objetiva y subjetivamente, no se observa una incidencia importante del programa Hábitat en el nivel de bienestar de los hogares en condición de pobreza extrema.

Umán: Desde el punto de vista de los usuarios del programa Hábitat a nivel personal, se muestra que 55 personas $(62.5 \%)$ reportan que es la primera vez que utilizan los servicios del programa, 15 (17.1\%) consideran que aprender es el beneficio obtenido, 8 (9.1\%) no respondió y 7 personas $(7.9 \%)$ consideran que ayuda a la superación personal y familiar. En relación con la participación dentro del programa, se tiene que 44 personas (50\%) es la primera vez que participan; 19 (21.6\%) piensan que esperan un mejor futuro; mientras que 14 (15.9\%) expresan que aprenden nuevas capacidades. Sobre el nivel de lo visto en los cursos los resultados son los mismos que para Kanasín.

Se concluye que, las condiciones objetivas de vida en Umán, son superiores a las de Kanasín, pero subjetivamente la población plantea mayores necesidades. En cuanto al programa Hábitat en el nivel de bienestar de los hogares en condición de pobreza extrema en habitabilidad es posible advertir cambios.

\subsection{Accesibilidad laboral}

Kanasín: En cuanto a la percepción de accesibilidad laboral en el mejoramiento del empleo, se tiene que del total de beneficiarios del programa es posible advertir que sólo $24 \%$ registra cambios en los últimos cinco años en la percepción salarial. Sin embargo, este cambio de salario no refleja una mejora sustancial en el ingreso de los participantes, ya que $47 \%$ percibe hasta dos salarios mínimos, es decir, se encuentran en condición de pobreza extrema, por lo que el espectro de pobreza de capacidades sigue siendo importante y no registra un peso importante en la disminución de la pobreza patrimonial; lo que permite sugerir la importancia de crear un mecanismo de enlace para crear espacios laborales y de autoempleo para los usuarios del programa Hábitat en su dimensión cursos. 
Se registró el punto de vista de las usuarias sobre el programa en el salario o en el empleo y los resultados muestran que 74 personas $(86.1 \%)$ consideran que si hay un impacto sobre el salario o empleo y tres personas $(3.5 \%)$ no consideran que el programa impacte.

Umán: el instrumento cualitativo registró el punto de vista de la población sobre el tiempo de traslado de los miembros de la familia al trabajo, a fin de encontrar correspondencia se tiene que 40 personas $(45.5 \%)$ declaran que ocupan menos de media hora; 18 (20.5\%) media hora; 14 (15.9\%) una hora y 10 personas $(11.4 \%)$ más de una hora. Se puede concluir que, el promedio de tiempo de traslado, es menor a una hora, lo que nos expresa una oferta laboral interna y externa del municipio, así como una buena infraestructura de comunicaciones.

\subsection{Participación social}

Kanasín: Se indaga sobre la percepción de la población en la organización o participación en las colonias. Los resultados muestran que 76 personas $(88.4 \%)$ consideran que si hay participación; no obstante, la entrevista grupal nos ofrece que la mayor parte de las usuarias no conoce ni está enterada de los espacios de gestión y reflexión del programa. Señalan que, además, la participación de la gente es muy escasa por la falta de información y contacto de las autoridades con la población y los canales de divulgación que utilizan son muy restringidos.

Umán: Para este municipio los resultados muestran que 66 personas (75\%) consideran que si hay una participación. Se puede apreciar que, la mayoría de los usuarios, consideran que el programa tiene un impacto en la participación y organización de la población. Sin embargo, en la entrevista grupal arroja que la mayor parte de las usuarias no conoce ni está enterada de los espacios de gestión y reflexión del programa; señala que la participación de la gente es escasa por falta de información y canales de divulgación. 


\section{CONCLUSIONES}

A nivel colectivo fue posible apreciar mediante el estudio realizado una mejora en el nivel de habitabilidad en los barrios, principalmente en términos de la infraestructura básica disponible y con la que se ha dotado a la población a través del programa. No obstante, la persistencia marcada por los problemas sociales y la carencia o insuficiencia de infraestructura básica son un claro indicador de la necesidad de seguir impulsando políticas complementarias y paralelas de atención a la población más vulnerable, para lo cual se debe continuar utilizando y mejorando la infraestructura ya creada hasta hoy, como es el caso de los centros comunitarios, lo cual resulta válido para los dos municipios estudiados. Resulta apremiante realizar acciones en el municipio de Kanasín, en donde se pudo comprobar que existen mayores necesidades humanas.

A nivel individual y familiar se logró constatar que los aprendizajes de conocimientos y técnicas aplicables a la vida cotidiana mejoran la capacidad de sobrevivencia de las familias, en el caso específico de las mujeres se crea un espacio para mejorar la autoestima y el reconocimiento de habilidades y destrezas para sí mismas y para compartir con la familia. Los conocimientos proporcionados constituyen herramientas para contribuir a obtener satisfactores antes inalcanzables, como es la confección de ropa, el bordado y urdido de hamacas, así como la repostería.

Diversas actividades productivas les han permitido mejorar su economía y contribuir al bienestar familiar, pues les ayuda a reducir algunos gastos y, en otros casos, a percibir algunos ingresos que, aunque son modestos, impulsan el bienestar familiar. Sería recomendable estructurar planes de apoyo que permitan otorgar recursos de manera accesible para que las familias tengan la posibilidad de formar un negocio propio.

Se considera también necesario eslabonar otros programas de desarrollo social de SEDESOL a fin de incidir en el abatimiento de la pobreza y la equidad de género, ya que a partir de los datos obtenidos no es posible dar cuenta de cambios importantes en los niveles de pobreza.

Los municipios estudiados presentan condiciones de operación del programa Hábitat distintas, que se traducen en mayores beneficios y con servicios de calidad a los usuarios de Umán, lo cual es acorde y en relación con los recursos invertidos. 
También se puede observar con claridad en las encuestas realizadas que el punto de vista de la población sobre el programa resulta ser positivo, aun cuando se observa un marcado desconocimiento del mismo y escasa participación. Por lo que se asume que, pese a los intentos de las autoridades, el programa se vislumbra sustancialmente de corte vertical, y se debe instrumentar una dinámica de transversalidad entre las comunidades involucradas que busque una mayor participación social en el diseño de las políticas de desarrollo.

\section{BIBLIOGRAFÍA}

Accinelli, E., J. Brida, L. Plata, y M. Puchet. 2008. "Bienestar social óptimos de Pareto y equilibrios Walrasianos", El Trimestre Económico, vol. LXXV, pp. 125-133, en http://ehis.ebscohost.com/eds/pdfviewer/pdfviewer?vid=2\&hid=109\& $\mathrm{sid}=\mathrm{a} 8583696-\mathrm{c} 928-4 \mathrm{c} 46-\mathrm{a} 203-\mathrm{e} 6 \mathrm{bb} 303 \mathrm{f7} \mathrm{c} 0 \mathrm{a} \% 40$ sessionmgr 110

Conapo. 2001. Índice de marginación a nivel localidad 2000, en http:// www.conapo.gob.mx/es/CONAPO/Indice_de_marginacion_a_nivel_localidad_2000

--------. 2012. Índice de marginación por entidad federativa y municipio 2010 , en http://www.conapo.gob.mx/es/CONAPO/Indices_de_Marginacion_2010_por_entidad_federativa_y_municipio

Coneval. 2005. Mapas de pobreza, 2005 por municipios en Yucatán, en http://www.coneval.gob.mx/cmsconeval/rw/pages/medicion/mapas/mapas2005.es.do;jsessionid $=69339 \mathrm{e} 325 \mathrm{~b} 9 \mathrm{e} 2605 \mathrm{eab} 24 \mathrm{~d} 81098 \mathrm{e} 5 \mathrm{e} 0 \mathrm{e} 66 \mathrm{c} 5062$ 49b18eb7e8f4018b0edc325d4.e34QaN4LaxeOa40Qaxf0 
Conde, J., C. Ocaña y G. Pérez. 2007. Análisis cuantitativo del estado de bienestar en Europa: modelos y resultados, Fundación de Estudios de Economía Aplicada. Documento de trabajo 2007-03, en: http://www.fedea.es/ pub/Papers/2007/dt2007-03.pdf

Chávez, J., H. Villareal, R. Cantú y H. González. 2009. "Efecto del incremento en el precio de los alimentos en la pobreza en México", El Trimestre Económico, vol. LXXVI, núm. 303, pp. 775-805, en: http://web. ebscohost. com/ehost $/$ pdfviewer/pdfviewer?vid $=3 \&$ hid $=24 \& \operatorname{sid}=57 \mathrm{~b} 3 \mathrm{c}$ 7e6-8635-4ad5-bf1e-b3de317d7a9f\%40sessionmgr10

Díaz, E. y E. Turner. 2012. "Pobreza y política social en México y estados de la frontera norte", Análisis Económico, vol. XXVII, núm. 64, en http:// www.analisiseconomico.com.mx/pdf/6403.pdf

Gamboa, L. y D. Cortez. 1999. Una discusión en torno al concepto de bienestar, Economía, Serie Documentos, Borradores de investigación, núm 1, en http://reocities.com/pjabad/sencolombiati.pdf

INEGI. 2000. Censo de población y vivienda. Resultados definitivos, CD.

-------. 2005. Conteo de población y vivienda. Resultados definitivos, CD.

-------. 2010. Censo de población y vivienda, 2010, Resultados definitivos, CD.

Navarro, T. 2011. "Aproximación a la evolución del estado de bienestar en España: Límites y oportunidades”, Contribuciones a las Ciencias Sociales, en http://www.eumed.net/rev/cccss/13/tnv.htm

POETY. 2006. Programa de ordenamiento ecológico territorial del estado de Yucatán, en http:/www.bitacoraordenamiento.yucatan.gob.mx/documentos/index.php?IdOrdenamiento $=2 \& I d U g a=\&$ Tipo $=$ Indicadores 
Presidencia de la República. 2007. Plan Nacional de Desarrollo, 2007-2012, México, http://pnd.calderon.presidencia.gob.mx/index. php?page=documentos-pdf

Secretaría de Desarrollo Rural y Pesca. 2001. Plan de desarrollo urbano de Umán, 2001 - 2004, Gobierno del estado de Yucatán, en http://www. campoyucatan.gob.mx/OEIDRUS/Municipalizacion/178_Merida/Uman/ Plan_Mpal_Uman.pdf

Ruiz-Castillo, J. 1999. Desigualdad y bienestar en España en términos reales, en http://eprints.ucm.es/6652/1/9808.pdf

SEDESOL. 2005. Evaluación Externa del Programa Hábitat. El Colegio de la Frontera Norte y SEDESOL. En http://www.2006-2012.sedesol.gob. $\mathrm{mx} /$ work/models/SEDESOL/Resource/1655/1/images/evaluacion_cobertura.pdf

SEDESOL. 2006. Primer informe de labores, en http://www.sedesol.gob. $\mathrm{mx} /$ work/models/SEDESOL/Resource/2140/I_InformeLabores2007SEDESOL.pdf

-------. 2008. Cuarto informe trimestral 2008, en http://www.sedesol.gob. $\mathrm{mx} /$ work/models/SEDESOL/Resource/1558/1/images/Cuarto_Informe_ Trimestral_2008.pdf

------. 2009. Cuarto informe trimestral 2009, en http://www.sedesol.gob. $\mathrm{mx} /$ work/models/SEDESOL/Resource/1558/1/images/Cuarto_Informe_ Trimestral_2009.pdf

-------. 2012. Página oficial de la Secretaría de Desarrollo Social, en http:// www.sedesol.gob.mx/es/SEDESOL/Habitat_Transparencia 